\title{
User Interaction Techniques for Future Lighting Systems
}

\author{
Dzmitry Aliakseyeu ${ }^{1}$, Jon Mason ${ }^{1}$, Bernt Meerbeek ${ }^{1}$, Harm van Essen ${ }^{2}$, \\ Serge Offermans ${ }^{2}$, and Andrés Lucero ${ }^{3}$ \\ ${ }^{1}$ Philips Research Europe, 5656 Eindhoven, The Netherlands \\ ${ }^{2}$ TU Eindhoven, Industrial Design department, Eindhoven, The Netherlands \\ ${ }^{3}$ Nokia Research Center, Visiokatu 1, 33720 Tampere, Finland \\ \{dzmitry.aliskseeyeu, jon.mason, bernt.meerbeek\} @philips.com \\ \{h.a.v.essen, s.a.m.offermans\}@tue.nl, andres.lucero@nokia.com
}

\begin{abstract}
LED-based lighting systems have introduced radically new possibilities in the area of artificial lighting. Being physically small the LED can be positioned or embedded into luminaires, materials and even the very fabric of a building or environment. Together with new functionality and flexibility comes complexity; the simple light switch is not anymore sufficient to control our light. This workshop explores new ways of interacting with light. The goal is to define directions for new forms of user interaction that will be able to support the emerging LED-based lighting systems.
\end{abstract}

Keywords: Lighting, User Interaction, LED, Smart lighting.

\section{Introduction}

The Light Emitting Diode (LED) has caused a profound change within the lighting industry. This is due in part to the LED's key properties of being physically small, highly efficient, digitally controlled and soon, very cheap to manufacture. Being physically small the LED can be positioned or embedded into luminaires, materials and even the very fabric of a building or environment [1]. The price to pay for all this functionality and flexibility is complexity. In the past, the single light bulb was controlled using a single switch; on and off. LED-based lighting systems can easily consist of hundreds separate light sources, with each source having many individually controllable parameters including colour, intensity, and saturation. With this high complexity, end-users cannot be expected to fully control all aspects of the lighting system. One direction that is being explored is to enrich lighting systems with sensor networks that will enable automatic lighting control that is based on contextual information [2]. However in many situations, such as setting up an atmospheric light, an explicit user interaction will still be required. Moreover, as functionality and complexity of light systems grow, the mapping between the sensors data and the desired light outcome will become fuzzy and will require an explicit user interaction for fine tuning the outcome or for adjusting the mapping between sensor input and light output. Thirdly, explicit interaction can be desired to allow users to feel in control while interacting with intelligent lighting systems. The light switch therefore in many situations will need to be replaced by novel forms of interactions that offer 
richer interaction possibilities such as tangible, multi-touch, or gesture-based user interfaces. As proliferation of LED light continues, it becomes more important to go beyond scattered design efforts [2,3] and systematically study user interaction with emerging lighting systems. The goal of this workshop is to take the first steps in this direction.

\section{Goals of the Workshop}

The focus of this workshop is on formulating key research challenges for user interaction with future lighting systems, creating initial design guidelines, and proposing novel interaction techniques for these systems. The goals of the workshop:

1. Make a first step toward expanding the design space of interactive technologies to include new forms of decorative, ambient, and task lighting.

2. Identify key challenges of UI for controlling new forms of lighting systems.

3. Establish a link with existing interaction paradigms that can be (re-)used for control of future lighting systems.

During the workshop, we would like to address and discuss the following questions:

- What design opportunities for interactive technology exist in the context of the new forms of lighting?

- What forms and types of (existing) interaction are suited for emerging lighting systems (in particular tangible, gesture and multi-display interaction techniques)?

- What forms of interaction are best suited for a global control (e.g. atmosphere) and what for a point control (e.g. task lighting)?

- How to balance between explicit user control and internal system control?

- How to use a lighting infrastructure as ambient displays and how to combine it with its primary function i.e. illumination?

- What is the impact of the proposed interaction techniques for complex lighting systems in other domains? What is the generalizability of these techniques?

To address the workshop questions we are inviting researchers to submit position papers that discuss or present new forms of interaction techniques for lighting. This topic deals with the research and design of new forms of user interaction or adaptation of existing ones to emerging lighting systems. The topic should attract researchers and designers working on new forms of interaction, LED lighting, smart lighting systems and who are interested in exploring UI for new emerging types and forms of luminaires and lighting systems.

\section{References}

1. Price, C.: Light Fantastic. Digital Home Magazine (November 2003)

2. Bhardwaj, S., Ozcelebi, T., Lukkien, J.: Smart lighting using LED luminaries. In: Proc. of PERCOM Workshops, pp. 654-659. IEEE, Los Alamitos (2010)

3. Lucero, A., Lashina, T., Terken, J.: Reducing Complexity of Interaction with Advanced Bathroom Lighting at Home. I-COM, Oldenbourg 5(1), 34-40 (2006) 